Anne-Cécile Lamy-Joswiak

DOI: $10.4312 /$ vestnik.11.385-390

Faculté des lettres, Université de Ljubljana

Slovénie

anne.cecile.lamy-joswiak@ff.uni-lj.si

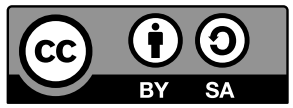

\title{
TENDANCES, MÉTHODE DE FRANÇAIS ET CAHIER D'ACTIVITÉS, NIVEAU C1/C2.
}

Liakin Denis, Liakina Natallia, Michaud Gabriel, Olivry Fabien. (2019). Tendances, méthode de français, niveau C1/C2. Paris : CLE International. ISBN : 978-209-038537-3. Broché, 208 pages, $24 €$.

Brito Amélie, Bucher Émilie. (2019). Tendances, méthode de français, niveau C1/C2. Cahier d'activités. Paris : CLE International. ISBN : 978-209-038538-0. Broché, 160 pages, $14 €$.

CLE International, maison d'édition spécialisée dans le français langue étrangère (FLE), publie enfin, depuis la parution du CECR (Cadre européen commun de référence pour les langues) en 2000 et sa large diffusion en 2001, sa première méthode d'apprentissage du FLE de niveau C1/C2, destinée aux adultes et grands adolescents. En effet, les seuls ouvrages conçus par CLE jusqu'à ce jour (et depuis 2007) pour ce niveau d'utilisateur expérimenté de la langue, proposaient des activités de préparation au DALF ${ }^{1}$ ou permettant d'acquérir les compétences correspondant à ce niveau défini par le $\mathrm{CECR}^{2}$, ou encore des outils complémentaires pour perfectionner la grammaire, le vocabulaire ou la communication dans la collection Progressive $d u$ français, destinée aux grands adolescents et adultes. Ainsi, la maison vient combler le vide de sa ligne éditoriale et entre en concurrence avec Hachette FLE et Didier, qui avaient respectivement publié leurs premières méthodes de niveaux C1/C2 en 2010 (Alter Ego 5) et 2018 (Édito C1).

Coordonnée par Jacques Pécheur et Jacky Girardet, illustre tandem de concepteurs des méthodes actionnelles Campus et Echo, la collection Tendances se voit désormais déclinée à tous les niveaux du Cadre. Les auteurs du niveau C1/C2, Denis Liakin,

1 Bourbon Vanessa, Chenard Samuelle, Lescure Richard, Mubanga Beya Anna, Rausch Alain, Vey Pauline, 2007. Nouveau DALF C1/C2 : 250 activités. 288 p. Barrière Isabelle, Parizet Marie-Louise, 2019. Abc DALF C1/C2:150 exercices (nouvelle édition). $328 \mathrm{p}$.

2 Kober-Kleinert Corinne, Parizet Marie-Louise, Poisson-Quinton Sylvie, 2007. Activités pour le Cadre européen commun de référence, niveaux C1/C2. $240 \mathrm{p}$. 
Natallia Liakina, Gabriel Michaud et Fabien Olivry, enseignent le français langue seconde et étrangère dans deux universités québécoises (Concordia et Mc Gill) et certains d'entre eux avaient déjà participé à la conception de la méthode Écho $A 1$ et $A 2$ avec Pécheur et Girardet, en adaptant celle-ci au public nord-américain. On retrouve donc, dans cette collection et notamment au niveau $\mathrm{C} 1 / \mathrm{C} 2$, l'approche actionnelle axée, selon les auteurs, autour de « scénarios en lien avec la réalité et les nouvelles pratiques sociales d'un environnement culturel francophone vaste et complexe, reflet des tendances globales actuelles ».

Le manuel comprend un livre de l'élève, assorti d'un cédérom contenant toutes les ressources complémentaires audio et vidéo, et un cahier d'activités. Il existe également en deux versions numériques payantes sur le site internet de l'éditeur : l'une destinée aux apprenants (Version numérique élève), l'autre aux enseignants (Version numérique enseignant), qui pourront l'utiliser par vidéoprojection et sur le TBI (tableau blanc interactif).

\section{Structure et contenus du livre de l'élève}

Le livre de l'élève est un ouvrage épais de plus de 200 pages dont une trentaine d'annexes contenant les transcriptions des documents audio et vidéos des leçons, et trois cartes illustrées représentant la France physique et touristique, la France administrative et le monde de la francophonie. Le tableau des contenus, réparti sur 4 pages, présente l'organisation de la méthode en 6 unités de 3 leçons chacune. Les 5 entrées du tableau intitulées objectifs actionnels, thèmes, actes de communication, civilisation, projet confirment la perspective résolument actionnelle de la méthode qui s'articule autour de 18 leçons ou « scénarios ».

La méthode est divisée en 6 grands dossiers (ou Unités) thématiques qui se succèdent selon la trame suivante : S'informer, Comprendre et expliquer le monde, Réfléchir aux réalités politiques et sociales, Consommer responsable, Entrer dans le monde du travail, S'intéresser à la culture. Ces intitulés inscrivent Tendances $C 1 / C 2$ dans une perspective d'éducation interculturelle et citoyenne par l'apprentissage des langues. Les 18 scénarios qui composent les 3 leçons de chaque unité visent à stimuler la réflexion en développant les compétences de savoir-faire et de savoir-être au monde contemporain.

Le thème de l'unité, les objectifs actionnels (ou tâches intermédiaires) et le projet apparaissent sur une double-page illustrée pour inaugurer chaque leçon. Si l'on prend l'exemple de la page inaugurale de l'Unité 1, Leçon 1, s'affichent les objectifs suivants : Se documenter à l'ère des fausse nouvelles (titre de la leçon), S'informer, Formuler une problématique, Évaluer les sources d'information, Se documenter efficacement (objectifs actionnels), Effectuer une recherche d'information (projet). Les contenus de civilisation, de grammaire et de vocabulaire ne font pas l'objet de pages spécifiques dans le livre ; d'ailleurs, ces deux derniers n'apparaissent même pas dans le tableau des contenus. Ils sont traités de façon transversale et en contexte dans des documents retenus pour leur 
nature, leur qualité et leur adéquation avec les niveaux $\mathrm{C} 1 / \mathrm{C} 2$, et font l'objet d'exercices systématiques dans le cahier d'activités.

Chaque leçon ou « scénario actionnel » se déroule sur 6 ou 8 pages, commence toujours avec un encadré déclencheur de réflexion sur la thématique, par le biais de questions ouvertes, propose un ensemble fourni de documents authentiques suivis d'activités de compréhension et de médiation, se poursuit avec la réalisation du projet, et se termine tantôt par une page Pause culture à la fin des leçons 1 et 2, tantôt par une double-page de préparation au DALF C1/C2 à la fin de la leçon 3 et pour clore l'unité. Qu'ils soient culturels, civilisationnels ou visant la préparation à l'examen (tâches de production orale $\mathrm{C} 1$ ou de compréhension/production écrites $\mathrm{C} 2$ ), ces contenus sont toujours en lien avec les documents traités en amont, dans la leçon.

Chaque scénario est structuré en tâches intermédiaires verbales, écrite ou orales, qui concourent à la réalisation du projet en fin de leçon. Celles-ci ne sont pas nécessairement calées sur une double-page, comme c'est le cas dans la majorité des méthodes. En effet, faire une synthèse nécessite un traitement de plus de deux pages ; par ailleurs, certains scénarios comme mener une enquête sur un enjeu de société ou écrire une lettre à un député peuvent comporter plus de trois étapes. En tous cas, les tâches s'appuient sur des documents authentiques, écrits ou oraux, suivis généralement de questions de compréhension, de reformulation ou de réflexion, et sont ponctuées d'activités de médiation entre pairs afin de préparer le projet, qu'il soit individuel ou collectif.

Des outils complémentaires balisent les leçons en fonction des compétences à acquérir et des tâches à réaliser : des encarts "Savoir-faire » proposent des outils méthodologiques pour, par exemple, rédiger une synthèse, prendre des notes, avancer des arguments, varier les formulations ou encore décrire un personnage de théâtre ; des " Points infos » à dimension socioculturelle développent des sujets très précis, comme par exemple l'obsolescence programmée, l'Office québécois de la langue française, la convention de stage, les principaux motifs de refus de candidature; des encadrés « Mieux s'exprimer » guident l'apprenant.e avec des expressions idiomatiques en vue de rapporter des propos, rédiger une lettre, analyser une infographie ou nuancer son point de vue, entre autres; et enfin, des encadrés grammaticaux mettent l'accent sur des points de grammaire particulièrement problématiques à un niveau avancé, notamment les structures avec quel, la mise en relation des idées, l'accord des noms collectifs et des expressions de quantité, la suffixation.

Les thématiques abordées dans Tendances $C 1 / C 2$ portent sur de nombreux sujets de société actuels comme autant de questions incitant à poser un regard critique et «citoyen » sur l'avenir : infox ou fausses nouvelles, gentrification, bilinguisme, écriture inclusive, légalisation du cannabis, intelligence artificielle, influenceurs, populisme, scandales environnementaux, obsolescence programmée, monnaies locales, Netflix et le cinéma, la décolonisation de la francophonie... Outre ces questions de réflexion sur le monde contemporain, certaines unités abordent des problématiques ancrées à la réalité 
des jeunes adultes pour qui cette méthode a été notamment conçue. Intitulée « Entrer dans le monde du travail », l'Unité 5, par exemple, est entièrement consacrée à la sphère professionnelle en insistant sur trois étapes : chercher un emploi, passer un entretien d'embauche, faire un stage en entreprise. Le scénario actionnel prévoit méthodiquement la rédaction d'un $\mathrm{CV}$, d'une lettre de motivation (leçon 1), la préparation de l'entretien professionnel (leçon 2), la rédaction du rapport de stage (leçon 3 ) mais entrevoit également les défis de la vie active : rejeter et accepter une candidature, présenter son parcours professionnel, revendiquer ses droits en contexte professionnel.

Transversale à la méthode en tant que compétence, la culture fait cependant l'objet d'une unité thématique (6) à part entière - « S'intéresser aux réalisations culturelles » s'inscrivant dans la perspective des trois nouvelles échelles du Volume complémentaire avec de nouveaux descripteurs $(2018)^{3}$ traitant du texte créatif et de la littérature. Cette thématique suit une trame actionnelle en trois temps ou leçons : commenter une fiction (roman, film, téléfilm, pièce de théâtre), commenter une œuvre d'art (art plastique, photo, architecture, jardins, vêtements...), faire le compte rendu d'une intervention orale ou écrite (article, intervention radiophonique, conférence, etc.). Parmi les tâches de production écrite et orale, il est proposé entre autres de réécrire un texte littéraire en français populaire contemporain, rédiger une fiche sur un personnage (leçon 1), présenter un tableau, défendre une œuvre d'art (leçon 2), rapporter le contenu d'une intervention sur un enjeu socioculturel (« Cannes contre Netflix, combat d'arrière-garde ?», leçon 3).

Les auteurs ont puisé dans des sources médiatiques francophones variées - Belgique, Cameroun, Canada, France, Suisse, entre autres - en créant un vaste répertoire de documents authentiques : émissions radiophoniques, reportages vidéos, articles de presse générale et spécialisée, document officiels et institutionnels, reproductions artistiques, infographies, rapports, enquêtes, critiques de cinéma, extraits littéraires... Cette gamme de supports hétéroclites invite à regarder et penser le monde contemporain, non plus depuis la France, mais en langue française à partir de pays francophones culturellement différents. Cette décentration du regard contribue au développement des compétences plurilingue et pluriculturelle et de la compétence de médiation préconisées notamment par les auteurs du CECR dans le Volume complémentaire (2018) ${ }^{4}$.

\section{Le cahier d'activités, un complément indispensable}

Le cahier d'activités suit scrupuleusement la progression du livre selon une organisation rigoureuse, et approfondit la compréhension des thématiques et l'acquisition des compétences communicatives langagières. Ainsi, pour chaque leçon apparaissent

3 https://rm.coe.int/cecr-volume-complementaire-avec-de-nouveaux-descripteurs/16807875d5 Voir en particulier p.119-122 pour ces trois échelles : Prendre des notes (conférences, séminaires, réunions) ; Exprimer une réaction personnelle à l'égard des textes créatifs (incluant la littérature); Analyser et critiquer des textes créatifs (incluant la littérature).

4 Voir p. 106 pour la compétence de médiation et p.164 pour la compétence plurilingue et pluriculturelle. 
successivement des activités de compréhension écrite, de vocabulaire, de grammaire et des outils méthodologiques visant la tâche de production écrite concluant l'unité, laquelle se termine par deux double-pages de bilan avec des activités de production écrite et orale, suivies d'outils culturels, souvent humoristiques (article sur l'humoriste Blanche Gardin, Unité 2 ou planches de BD de Pénélope Bagieu, Unité 4 et de Franquin, Unité 5) sinon historiques (débuts du journalisme en France, Unité 1) ou sociologiques (portrait et chanson de Gaël Faye, Unité 6). Le cahier contient également 4 épreuves d'entraînement au DALF, trois visant le $\mathrm{C} 1$ et une le $\mathrm{C} 2$, respectivement aux termes des unités 2, 4 et 6 . Un livret est fourni en annexe avec les corrigés de toutes les activités du cahier ainsi que les transcriptions des documents audio des activités d'entraînement au DALF, ce qui facilite l'apprentissage en autonomie.

Le travail sur les compétences communicatives langagières étant absent du livre, le cahier d'activités s'avère, à ce niveau $\mathrm{C} 1 / \mathrm{C} 2$, un outil indispensable à l'enrichissement et au perfectionnement des compétences linguistiques (vocabulaire, grammaire, phonologie). Les activités de compréhension écrite et orale sont toujours détaillées, prolongeant les questions de compréhension globale présentes dans le livre de l'élève. Notons qu'elles adoptent la même typologie que celles soumises dans les épreuves du DALF (VRAI/ FAUX, QCM, QROC, QROL ${ }^{5}$ ). Quant aux activités lexicales, elles commencent toujours par l'apprentissage d'une liste de mots (noms, verbes, adjectifs, adverbes et locutions) relatifs à la thématique de l'unité. Celle-ci est suivie d'une série d'exercices (3/4) de complétion, de classement et d'appariement portant sur la synonymie, l'antonymie, l'hyperonymie, la reformulation. La grammaire est traitée de façon approfondie dans des exercices lacunaires, structuraux (de transformation ou de substitution) ou de transformation, et toujours en contexte. À ce niveau, les auteures du cahier ont choisi de faire réviser et perfectionner la maîtrise des modes et des temps verbaux, des connecteurs logiques, l'interrogation, la mise en relief, la nominalisation, la pronominalisation, le passif, les pronoms relatifs simples et composés, le comparatif, l'accord de noms collectifs et l'expression de la quantité. Le bilan de chaque unité contient des outils méthodologiques qui préparent graduellement à la réalisation des tâches de production écrite et orale en fin d'unité, et des outils culturels dont les supports textuels (faisant l'objet d'activités d'analyse et de compréhension) apportent un éclairage socioculturel original et récréatif sur la thématique abordée. La phonologie, et en particulier le travail de perfectionnement sur les traits prosodiques qui font souvent défaut à ce public de niveau avancé - varier l'intonation et placer correctement l'accent pour exprimer ce qu'on souhaite dire - constitue à notre avis, la grande absente des compétences langagières approfondies dans le cahier d'activités.

$5 \mathrm{QCM}=$ question à choix multiple, $\mathrm{QROC}=$ question à réponse ouverte courte, $\mathrm{QROL}=$ question à réponse ouverte longue 


\section{Conclusion}

Si de prime abord, Tendances semble essentiellement axé sur la préparation au DALF $\mathrm{C} 1 / \mathrm{C} 2$ dont elle regorge d'exercices d'entraînements et d'épreuves types, l'analyse approfondie de la méthode démontre son fort potentiel d'utilisation par des enseignants de FLE, notamment friands de la perspective actionnelle, au niveau universitaire ou dans des centres de langue, ou encore par des apprenant.e.s autonomes. À défaut d'avoir testé la méthode in situ, on peut toutefois évaluer la durée d'exécution d'une unité thématique entre 20 et 30 heures en présentiel, tant les contenus sont denses, à condition toutefois que les tâches du cahier d'activités soient réalisées en autonomie. Les unités thématiques fonctionnent indépendamment les unes des autres et peuvent être traitées comme telles. Cela dit, l'Unité 1 («S'informer et étudier»), qui aborde le traitement des textes et des informations, nous semble pertinente sinon indispensable en position inaugurale car ses tâches intégratives fournissent des bases fondamentales aux compétences visées dans les unités suivantes. D'ailleurs, le travail approfondi d'analyse textuelle et stylistique est l'un des atouts de cette méthode. Face à un public professionnel aguerri, l'on pourra faire l'impasse sur l'Unité 5 («Entrer dans le monde du travail »), par exemple. Les quatre autres unités développent également une compétence que nous estimons indispensable à tout individu avide de langues et de culture, à savoir celle de "savoir être au monde contemporain », autrement dit une compétence citoyenne. L'organisation en double-page «prête à l'emploi », la sobriété de la maquette, les outils compagnons (DVD-Rom et livret de corrigés et de transcription), le choix de thématiques contemporaines facilitent l'enseignement/apprentissage, et l'enchaînement linéaire et compréhensible des leçons, ainsi que son caractère résolument francophone font de Tendances un outil méthodologique efficace en fonction des besoins du public visant les niveaux $\mathrm{C} 1 / \mathrm{C} 2$. 